\title{
Comprehensive study of instable regions in Pseudomonas aeruginosa and Mycobacterium tuberculosis
}

\author{
Dan Wang ${ }^{1}$, Jingyu $\mathrm{Li}^{1}$ and Lusheng Wang ${ }^{1,2^{*}}$
}

From 5th International Work-Conference on Bioinformatics and Biomedical Engineering Granada, Spain. 26-28 April 2017

\author{
${ }^{*}$ Correspondence: \\ cswangl@cityu.edu.hk \\ ${ }^{1}$ Department of Computer \\ Science, City University \\ of Hong Kong, 83 Tat Chee \\ Ave., Hong Kong, People's \\ Republic of China \\ Full list of author information \\ is available at the end of the \\ article
}

\begin{abstract}
Background: Pseudomonas aeruginosa is a common bacterium which is recognized for its association with hospital-acquired infections and its advanced antibiotic resistance mechanisms. Tuberculosis, one of the major causes of mortality, is initiated by the deposition of Mycobacterium tuberculosis. Accessory sequences shared by a subset of strains of a species play an important role in a species' evolution, antibiotic resistance and infectious potential.
\end{abstract}

Results: Here, with a multiple sequence aligner, we segmented 25 P. aeruginosa genomes and $28 \mathrm{M}$. tuberculosis genomes into core blocks (include sequences shared by all the input genomes) and dispensable blocks (include sequences shared by a subset of the input genomes), respectively. For each input genome, we then constructed a scaffold consisting of its core and dispensable blocks sorted by blocks' locations on the chromosomes. Consecutive dispensable blocks on these scaffold formed instable regions. After a comprehensive study of these instable regions, three characteristics of instable regions are summarized: instable regions were short, site specific and varied in different strains. Three DNA elements (directed repeats (DRs), transposons and integrons) were then studied to see whether these DNA elements are associated with the variation of instable regions. A pipeline was developed to search for DR pairs on the flank of every instable sequence. 27 DR pairs in P. aeruginosa strains and 6 pairs in M. tuberculosis strains were found to exist in the instable regions. On the average, $14 \%$ and $12 \%$ of instable regions in P. aeruginosa strains covered transposase genes and integrase genes, respectively. In M. tuberculosis strains, an average of $43 \%$ and $8 \%$ of instable regions contain transposase genes and integrase genes, respectively.

Conclusions: Instable regions were short, site specific and varied in different strains for both P. aeruginosa and M. tuberculosis. Our experimental results showed that DRs, transposons and integrons may be associated with variation of instable regions.

Keywords: Pan-genome, Dispensable genome, Insertion, Deletion, Homologous recombination, Directed repeats, Transposase, Integrase 


\section{Background}

Tettelin firstly coined the term pan-genome in his research of Streptococcus agalactiae [1] more than a decade ago. Pan-genome describes the union of genomes in a clade of interest, including core and dispensable genome. Core genome includes sequences shared by all genomes of interest while dispensable genome is the intersection of a subset of the genomes of interest. Core genome of a clade are typically responsible for the major phenotypic traits and basic aspects of the biology of this clade while dispensable genome contributes to the species diversity and persistence in a particular environment [2]. Identification and study of dispensable genome is essential for better understanding of a species' evolution, niches adaptation, antibiotic resistance, infectious potential and colonization of a new host. Pan-genome of closely related strains can be obtained at the nucleotide sequence level using multiple whole-genome alignment tools.

Bacterial genomes are dynamic on the evolutionary time scale. The existence of dispensable genome of a bacterial species may arise from genome rearrangement events such as insertions or deletions (indels), or from the activities of mobile DNA elements such as transposons or integrons.

Several previous studies show that indels can be mediated by directed repeats (DRs) [3, 4]. And these DR-mediated indel events are named homologous recombination in which DNA strands are exchanged between a pair of similar or identical sequences. Sequences flanked by a pair of DRs can be deleted from the chromosomes or be inserted into new chromosomes, for example, numerous horizontally transferred genes are integrated into their new host chromosomes through homologous recombination [5]. The estimated minimal length of homologous sequences which are necessary for the recombination process is between 20 and $100 \mathrm{bp}$ [6].

Existence of dispensable genome can also result from the activities of mobile DNA elements, such as transposons and integrons. There are two major ways for transposable genetic elements to move from one locus to another within or between genomes. One involves passage through an RNA intermediate prior to synthesis of a DNA copy while the other is limited uniquely to DNA intermediates. For both types of elements, recombination reactions involved in integration are carried out by element-specific enzymes. These are called transposase (Tnp) in the case of DNA elements and integrase in the case of the best-characterized RNA elements, the retroviruses and retrotransposons [7].

In our study, we analyzed 25 Pseudomonas aeruginosa and 28 Mycobacterium tuberculosis complete genomes downloaded from the NCBI GenBank database, respectively. Genomic sequences were segmented into core blocks (include sequences shared by all genomes) and dispensable blocks (include sequences shared by a subset of all genomes) by using a multiple sequence aligner named Mugsy [8]. Consecutive dispensable blocks flanked by a pair of core blocks formed an instable region (InsR) and this pair of core blocks are the insertion site for this instable region. DNA sequences within a instable region are called instable sequences. For the $28 \mathrm{M}$. tuberculosis strains (see Additional file 1: Table S5 for more details), we conducted the same experiment as P. aeruginosa strains and achieved $28 \mathrm{M}$. tuberculosis scaffolds. 


\section{Results}

In this section, we showed three characteristics of instable regions and illustrated two kinds of mechanisms for the insertion and deletion of instable regions based on $P$. aeruginosa and M. tuberculosis genomes.

\section{Characteristics of instable regions}

We conducted a comprehensive study on instable regions in both $P$. aeruginosa and $M$. tuberculosis strains and found three characteristics of instable regions: instable regions were short, site specific and varied in different strains.

\section{Instable regions are short}

Among the $25 P$. aeruginosa strains, the number of instable regions was from 44 to 57 and the total lengths of instable regions were from 691,941 to 1,794,274 bps which accounted for $11 \%$ to $24 \%$ of their respective whole genome (see Additional file 1: Table S2). There were a total of 1231 instable regions among the 25 strains. The median and average length of these 1231 regions were $6.6 \mathrm{kbps}$ and $20.9 \mathrm{kbps}$, respectively. The histogram for the length (in bp) of these 1231 instable regions is in Fig. 1a. We found that $40.2 \%$ of instable regions were shorter than $5 \mathrm{kbp}, 77.7 \%$ of instable regions were shorter than $20 \mathrm{kbp}$ and only $2.6 \%$ of instable regions are longer than $120 \mathrm{kbp}$ (see Fig. 1a).

In the $28 \mathrm{M}$. tuberculosis strains, the total amount of instable regions varied from 18 to 29. The total lengths of instable regions were from 40,009 to 71,578 bp which accounted for $0.91 \%$ to $1.62 \%$ of their respective whole genome (see Additional file 1: Table S5). A total of 671 instable regions existed in the $28 \mathrm{M}$. tuberculosis strains. The median and average length of these 671 regions were $1300 \mathrm{bp}$ and $2343 \mathrm{bp}$, respectively. We plotted the histogram for the length (in bp) of these 671 instable regions (see Fig. 2a). 77.9\% of instable regions were shorter than $2 \mathrm{kbp}, 96.3 \%$ of instable regions were shorter than 10 kbp and only $1.04 \%$ of instable regions are longer than 15 kbp (see Fig. 2a).

\section{Instable regions are site-specific}

For 25 P. aeruginosa strains, 451 core blocks could be generated in the scaffolds. If the orders of these core blocks are consistent in the 25 scaffolds, there will be 450 distinct pairs

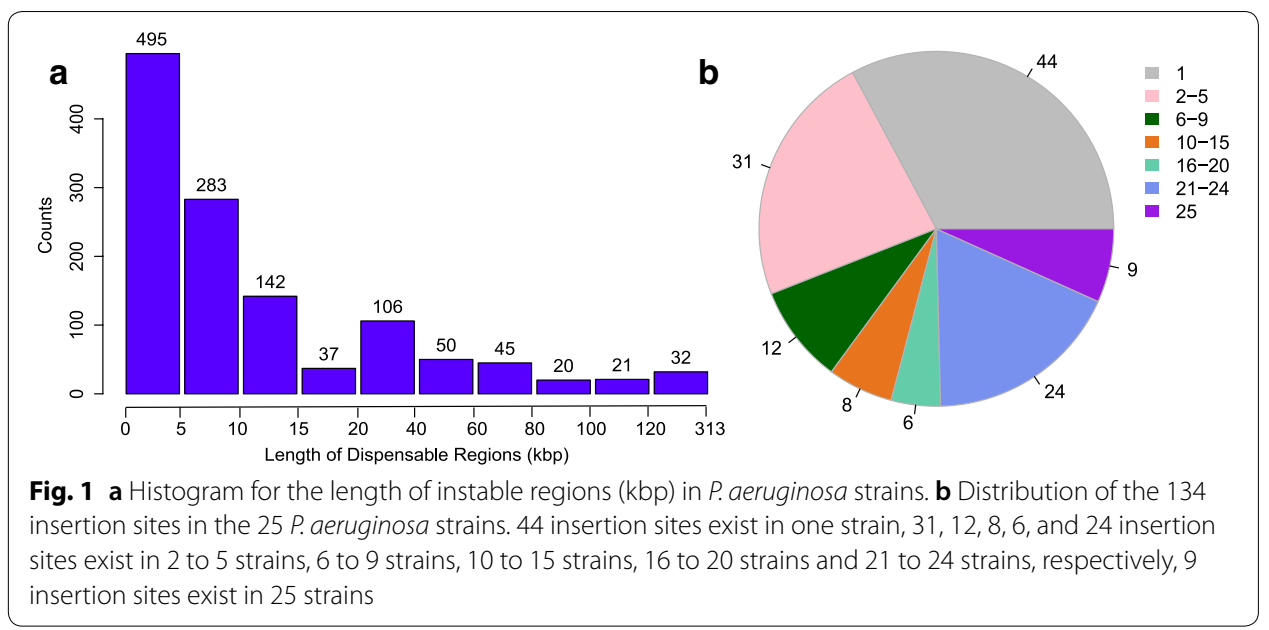




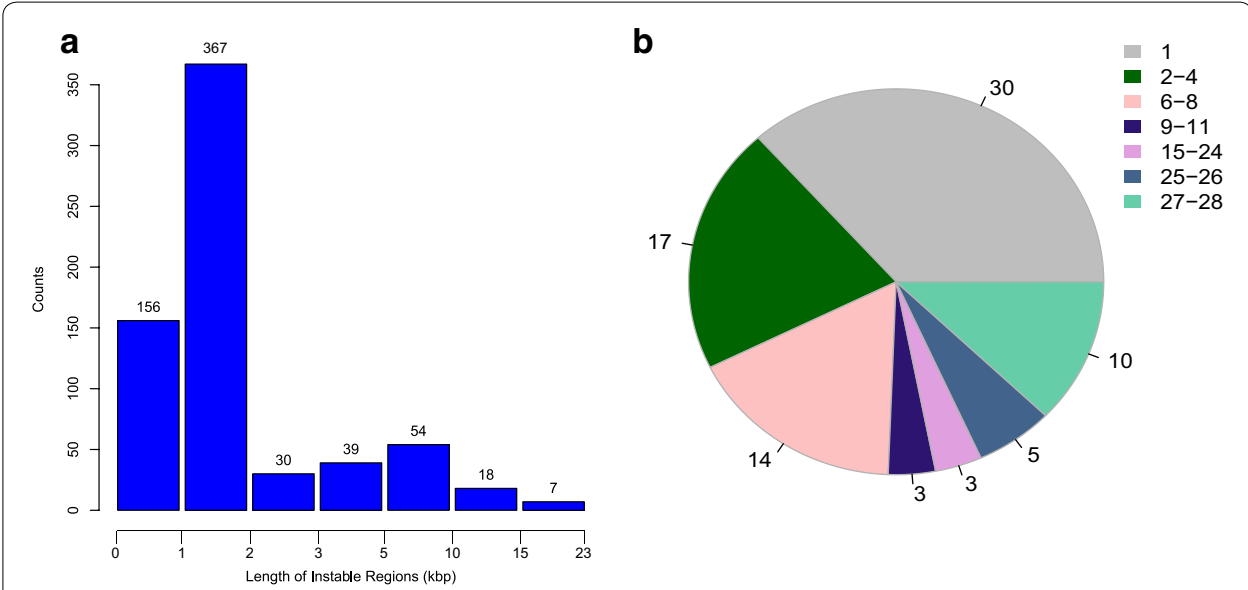

Fig. 2 a Histogram for the length of instable regions (kbp) in M. tuberculosis strains. $\mathbf{b}$ Distribution of the 82 insertion sites in the $28 \mathrm{M}$. tuberculosis strains. 30 insertion sites exist in one strain, 17, 14, 3, 3, 5 and 10 insertion sites exist in 2 to 4 strains, 6 to 8 strains, 9 to 11 strains, 15 to 24 strains, 25 to 26 strains and 27 to 28 strains, respectively

of adjacent core blocks which can be the potential insertion sites for instable regions. But in fact, the orders of core blocks were different in the 25 scaffolds, which may result from genome rearrangements. As a consequence, we found a total of 506 distinct pairs of adjacent core blocks which can be the potential insertion sites for instable regions. Among the 506 pairs of adjacent core blocks, 134 pairs of core blocks are found between which instable regions existed (the block IDs of these 134 pairs of core blocks are listed in Additional file 1: Table S3). For these 134 pairs of core blocks, 90 of them were insertion sites in two or more strains while 44 of them were insertion sites in only one strain (see Fig. 1b). There were 9 pairs of core blocks which were insertion sites in all the 25 strains (see Fig. 1b).

As for M. tuberculosis strains, there were 107 distinct pairs of adjacent core blocks which can be the potential insertion sites of instable regions. However, 82 pairs of core blocks between which instable regions really existed. Within the 82 pairs of core blocks, 52 insertion sites were in two or more strains while 30 existed in only one strain in all the $28 \mathrm{M}$. tuberculosis strains (see Additional file 1: Table S6).

\section{Instable regions vary in different strains}

Between the same pair of core blocks, the inserted instable region varied a lot in different strains. For example, between Block 8 and 14, the instable regions in the 25 P. aeruginosa strains were different (see Fig. 3). For $P$. aeruginosa strains, among the 90 insertion sites which existed in two or more strains, instable regions in 57 insertion sites were varied in different strains (the block IDs of these 57 insertion sites are in Additional file 1: Table S3). In $M$. tuberculosis strains, instable regions in 25 (out of 52) insertions sites are varied in different strains (see Additional file 1: Table S6).

\section{Mechanisms for the variation of instable regions}

Due to the large variation of instable regions, we focused on directed repeats (DRs), transposase genes and integrase genes. Those DNA elements may contribute to the variation of instable regions. 


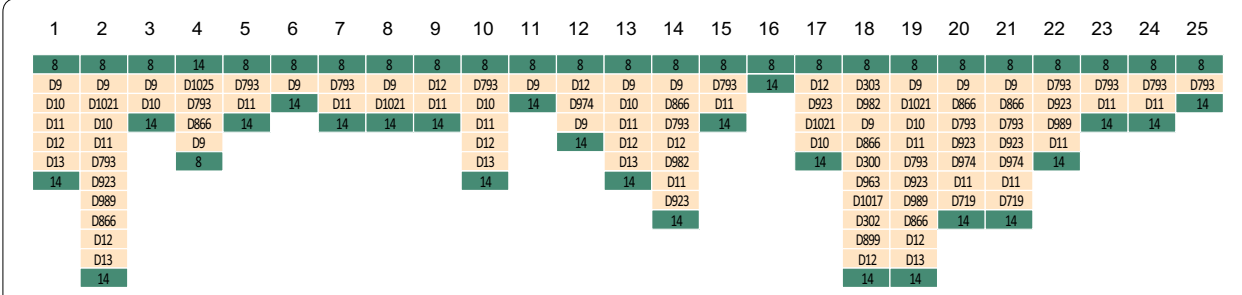

Fig. 3 Instable regions between Core Block 8 and 14 in the 25 P. aeruginosa strains. The ID of each strain is labeled above its respective column. Column $\mathrm{x}$ is the partial scaffold between Core Block 8 and 14 in Strain $x$, where $x=1,2, \ldots, 25$. Core blocks and dispensable blocks are respectively represented by green and yellow rectangular boxes with block IDs written on them

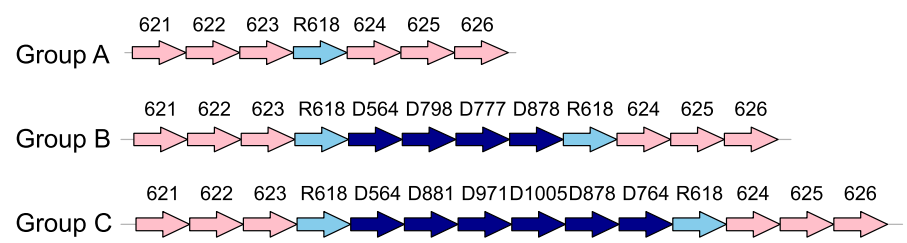

Fig. 4 Insertion of instable regions mediated by R618 in P. aeruginosa strains. The three rows are the partial scaffolds (from Block 621 to 626) of strains in Group A, B and C. Blocks are represented by arrows. Core blocks are in pink, dispensable blocks are in dark blue and Repeat R618 is in light blue. The number above an arrow is the ID of the corresponding block

\section{Indels mediated by directed repeats (homologous recombination)}

We developed a pipeline to find directed repeat pairs (for the explanation of DRs, see "Methods" section) which were on the flank of an instable sequence and applied it on the 25 P. aeruginosa strains and $28 M$. tuberculosis strains, respectively. After achieving DRs by using our pipeline, we added those DRs into the 25 P. aeruginosa scaffolds and $28 M$. tuberculosis scaffolds respectively according to DRs' positions in the chromosomes (see the scaffolds in Additional file 1: Tables S1 and S4, DRs are represented by R plus an ID number).

After studying the scaffolds with DRs, we found 27 pairs of DRs in P. aeruginosa strains and 6 pairs of DRs in M. tuberculosis strains existing in the instable regions.

Figure 4 one example pair of DRs found in P. aeruginosa which may mediate the change of the instable region between Core Block 623 and 624 (see Fig. 4 and the DR ID is R618). In Fig. 4, the 25 strains are divided into three groups (Group A, B, and C) according to their scaffolds between Core Block 623 and 624. Group A includes Strain 1, 3, 5, 7, 9, 10, 11, 12, 13, 15, 16, 17, 20, 21 and 25, Group B includes Strain 2, 4, 14, and 23 and Group C includes Strain 8 only. In the strains of Group A, there was only one copy of R618 between Block 623 and 624 while in the strains of Group B and C, there was an instable region flanked by a pair of R618 between Block 623 and 624 (see Fig. 4). The instable regions in Group B and $C$ were different: the instable region of Group B contained dispensable blocks D564, D798, D777 and D878 while the instable region of Group C contained D564, D881, D971, D1005, D878 and D764 (see Fig. 4). We believed the pair of R618 mediated the insertion of the instable regions in Group $B$ and $C$ because the existence of the two copies of R618 made sure the ends of Block 623 and 624 remained unchanged before and after the insertion of the instable region. 
For every achieved DR pair $R n$, we showed the 25 strains' partial scaffolds covering $R n$ in Additional file 1: Table $\mathrm{Rn}$ (where $\mathrm{n}$ is the ID of this DR pair).

Two example pairs of DRs (see Fig. 5 and DR IDs are R3 and R4) found in M. tuberculosis strains is demonstrated in Fig. 5. According to the existence of the blue and red regions, the $28 \mathrm{M}$. tuberculosis strains are divided into four groups (Group 1-4, see Fig. 5). Group 2 includes Strain 6 and 9, Group 3 includes Strain 21 and 28, Group 4 includes Strain 14 and Group 1 includes the remaining strains. The insertion or deletion of the blue region is mediated by $\mathrm{R} 3$ and that of the red region is mediated by R4.

The remaining DRs are shown in Additional file 1: Tables S1, S4 and Rn, where $\mathrm{n}$ is the ID of DR.

\section{Transposase and integrase genes}

We added the genes whose products are integrase and transposase into the scaffolds for all the strains except $P$. aeruginosa Strain 23, 15 and 24 which lacks annotation information (see the scaffolds in Additional file 1: Tables S1 and S4, TNP and IN represent transposase and integrase genes, respectively). We then further studied the correlations between these genes and instable regions. In $P$. aeruginosa strains, we found that more than $60 \%$ of integrase and transposase genes were located inside the instable regions in all the strains except Strain 25 (see Table 1, Column P1 and P2). In P. aeruginosa Strain $3,4,5,6,7,8,9,10,11,12,14,17,20$ and 21 , all genes which encode to be transposase were in instable regions and for Strain 20, 21 and 16, all integrase genes were inside the instable regions (see Table 1, Column P1 and P2). We found that, in $P$. aeruginosa strains, $2 \%-30 \%$ covered integrase genes (see Column P4 of Table 1 ) and $2 \%$ to $42 \%$ of instable regions covered transposase genes (see Column P3 of Table 1). On average, 92\% of transposase genes were within instable regions, while $85 \%$ of integrase genes were within instable regions, $14 \%$ of instable regions cover transposase genes and $12 \%$ of instable regions cover integrase genes in $P$. aeruginosa strains (see the last row in Table 1).

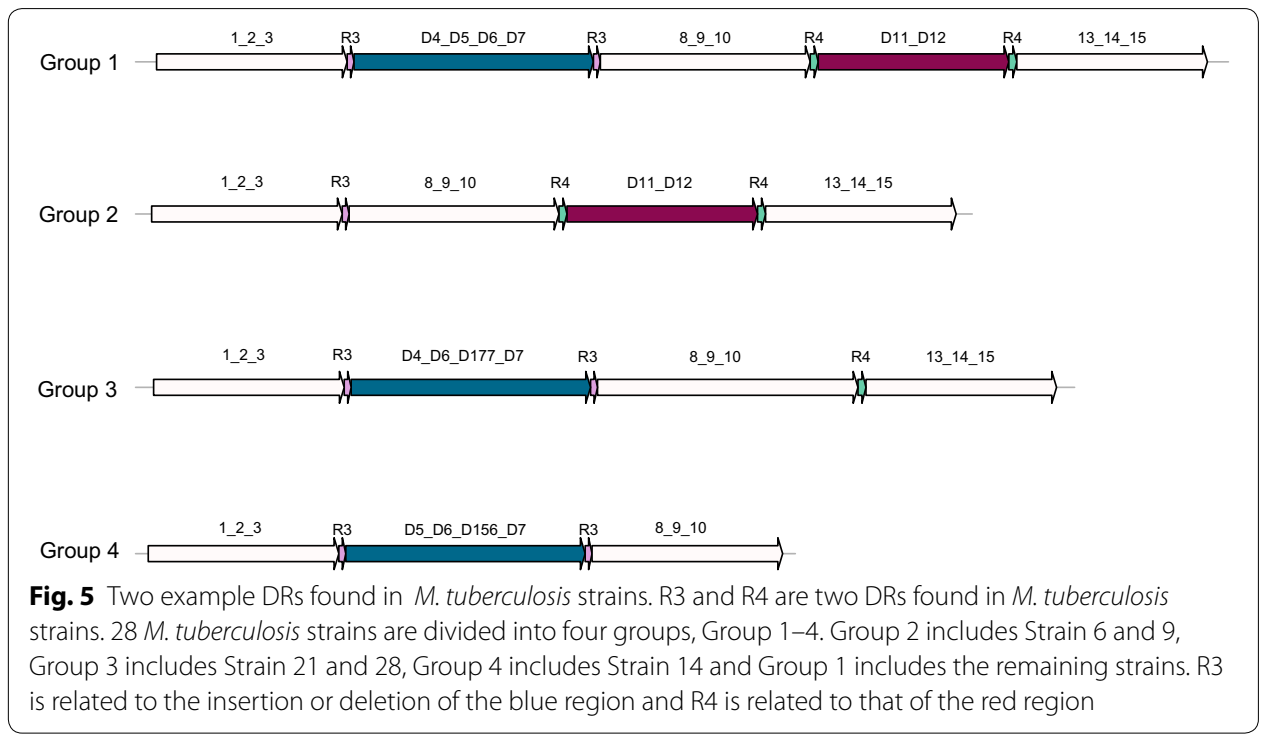


Table 1 Genes of transposase and integrase in instable regions of $P$. aeruginosa Strain 1 to 25

\begin{tabular}{|c|c|c|c|}
\hline P1* & P2* & P3* & P4* \\
\hline $81 \%(34 / 42)$ & $83 \%(10 / 12)$ & $27 \%(15 / 56)$ & $13 \%(7 / 56)$ \\
\hline $96 \%(48 / 50)$ & $92 \%(11 / 12)$ & $28 \%(14 / 50)$ & $14 \%(7 / 50)$ \\
\hline $100 \%(6 / 6)$ & $88 \%(7 / 8)$ & $11 \%(5 / 47)$ & $13 \%(6 / 47)$ \\
\hline $100 \%(17 / 17)$ & $67 \%(6 / 9)$ & $17 \%(9 / 52)$ & $12 \%(6 / 52)$ \\
\hline $100 \%(5 / 5)$ & $88 \%(7 / 8)$ & $9 \%(4 / 45)$ & $11 \%(5 / 45)$ \\
\hline $100 \%(1 / 1)$ & $71 \%(5 / 7)$ & $2 \%(1 / 48)$ & $10 \%(5 / 48)$ \\
\hline $100 \%(12 / 12)$ & $75 \%(3 / 4)$ & $16 \%(7 / 44)$ & $7 \%(3 / 44)$ \\
\hline $100 \%(2 / 2)$ & $83 \%(5 / 6)$ & $4 \%(2 / 52)$ & $8 \%(4 / 52)$ \\
\hline $100 \%(13 / 13)$ & $90 \%(9 / 10)$ & $13 \%(6 / 45)$ & $13 \%(6 / 45)$ \\
\hline $100 \%(25 / 25)$ & $89 \%(8 / 9)$ & $20 \%(11 / 54)$ & $11 \%(6 / 54)$ \\
\hline 100\% (8/8) & $88 \%(7 / 8)$ & $6 \%(3 / 51)$ & $14 \%(7 / 51)$ \\
\hline $100 \%(15 / 15)$ & $92 \%(12 / 13)$ & $9 \%(4 / 47)$ & $13 \%(6 / 47)$ \\
\hline $80 \%(32 / 40)$ & $83 \%(10 / 12)$ & $27 \%(15 / 56)$ & $13 \%(7 / 56)$ \\
\hline $100 \%(5 / 5)$ & $91 \%(10 / 11)$ & $9 \%(4 / 47)$ & $11 \%(5 / 47)$ \\
\hline $92 \%(11 / 12)$ & $100 \%(10 / 10)$ & $11 \%(5 / 47)$ & $13 \%(6 / 47)$ \\
\hline $100 \%$ (8/8) & $83 \%(5 / 6)$ & $11 \%(5 / 47)$ & $9 \%(4 / 47)$ \\
\hline $95 \%(21 / 22)$ & $84 \%(21 / 25)$ & $18 \%(8 / 45)$ & $24 \%(11 / 45)$ \\
\hline $86 \%(68 / 79)$ & $90 \%(26 / 29)$ & $42 \%(24 / 57)$ & $30 \%(17 / 57)$ \\
\hline $100 \%(1 / 1)$ & $100 \%(1 / 1)$ & $2 \%(1 / 45)$ & $2 \%(1 / 45)$ \\
\hline $100 \%(1 / 1)$ & $100 \%(1 / 1)$ & $2 \%(1 / 45)$ & $2 \%(1 / 45)$ \\
\hline $69 \%(40 / 58)$ & $82 \%(9 / 11)$ & $29 \%(14 / 49)$ & $14 \%(7 / 49)$ \\
\hline $17 \%(1 / 6)$ & $50 \%(1 / 2)$ & $2 \%(1 / 45)$ & $2 \%(1 / 45)$ \\
\hline $92 \%$ & $85 \%$ & $14 \%$ & $12 \%$ \\
\hline
\end{tabular}

$\mathrm{P} 1$ is the percentage of Tnp genes within InsRs (no. of Tnp in InsRs/total no. of Tnp). P2 is the percentage of IN genes within InsRs (no. of IN in InsRs/total no. of IN). P3 is the percentage of InsRs which covers Tnp genes (no. of InsRs which covers Tnp Genes/total no. of InsRs). P4 is the percentage of InsRs which covers IN genes (no. of InsRs which covers IN Genes/total no. of InsRs)

Table 2 shows the situations of integrase and transposase genes in instable regions of $M$. tuberculosis Strain 1 to 28. We found that in M. tuberculosis strains, on average, $45 \%$ of transposase genes were within instable regions and $28 \%$ of integrase genes were within instable regions. On average, $43 \%$ and $8 \%$ of instable regions in M. tuberculosis strains covered transposase and integrase genes respectively.

To sum up, we drew 28 scaffolds for $M$. tuberculosis strains and 25 scaffolds for P. aeruginosa strains. These scaffolds are composed by core blocks, dispensable blocks, transposase, directed repeats, and integrase genes and sorted by their positions in the strains' chromosomes (see Additional file 1: Tables S1 and S4). We plotted Figs. 6 and 7 to visualize the $P$. aeruginosa scaffolds and M. tuberculosis scaffolds respectively. From Figs. 6 and 7, we can see that transposase, directed repeats and integrase genes are in high correlation with instable regions.

\section{Methods}

In this section, we introduced how gene scaffolds for $P$. aeruginosa and $M$. tuberculosis strain sets are established and the pipeline details for finding DRs that will mediate insertion and deletion of instable blocks. In the end, we explained the method for finding DNA elements such as transposases and integrases. 
Table 2 Genes of transposase and integrase in instable regions of $M$. tuberculosis Strain 1 to 28

\begin{tabular}{|c|c|c|c|}
\hline $\mathrm{P} 1 *$ & P2* & P3* & P4* \\
\hline $42 \%(14 / 33)$ & $29 \%(2 / 7)$ & $35 \%(9 / 26)$ & $8 \%(2 / 26)$ \\
\hline $29 \%(10 / 35)$ & $17 \%(1 / 6)$ & $20 \%(5 / 25)$ & $4 \%(1 / 25)$ \\
\hline $40 \%(14 / 35)$ & $33 \%(2 / 6)$ & $21 \%(6 / 29)$ & $7 \%(2 / 29)$ \\
\hline $64 \%(32 / 50)$ & $14 \%(1 / 7)$ & $71 \%(17 / 24)$ & $4 \%(1 / 24)$ \\
\hline $34 \%(12 / 35)$ & $0 \%(0 / 6)$ & $21 \%(6 / 28)$ & $0 \%(0 / 28)$ \\
\hline $57 \%(29 / 51)$ & $17 \%(1 / 6)$ & $77 \%(20 / 26)$ & $4 \%(1 / 26)$ \\
\hline $35 \%(13 / 37)$ & $38 \%(3 / 8)$ & $32 \%(8 / 25)$ & $12 \%(3 / 25)$ \\
\hline $70 \%(30 / 43)$ & $43 \%(3 / 7)$ & $77 \%(17 / 22)$ & $9 \%(2 / 22)$ \\
\hline $33 \%(12 / 36)$ & $14 \%(1 / 7)$ & $24 \%(5 / 21)$ & $5 \%(1 / 21)$ \\
\hline $32 \%(11 / 34)$ & $25 \%(2 / 8)$ & $33 \%(6 / 18)$ & $11 \%(2 / 18)$ \\
\hline $35 \%(13 / 37)$ & $38 \%(3 / 8)$ & $31 \%(8 / 26)$ & $12 \%(3 / 26)$ \\
\hline $42 \%(15 / 36)$ & $67 \%(2 / 3)$ & $35 \%(9 / 26)$ & $8 \%(2 / 26)$ \\
\hline $33 \%(12 / 36)$ & $38 \%(3 / 8)$ & $30 \%(6 / 20)$ & $15 \%(3 / 20)$ \\
\hline $31 \%(11 / 36)$ & $14 \%(1 / 7)$ & $20 \%(4 / 20)$ & $5 \%(1 / 20)$ \\
\hline $65 \%(32 / 49)$ & $14 \%(1 / 7)$ & $67 \%(18 / 27)$ & $4 \%(1 / 27)$ \\
\hline $45 \%(20 / 44)$ & $38 \%(3 / 8)$ & $59 \%(13 / 22)$ & $14 \%(3 / 22)$ \\
\hline $72 \%(33 / 46)$ & $43 \%(3 / 7)$ & $78 \%(18 / 23)$ & $9 \%(2 / 23)$ \\
\hline $41 \%(14 / 34)$ & $17 \%(1 / 6)$ & $24 \%(6 / 25)$ & $4 \%(1 / 25)$ \\
\hline $40 \%(14 / 35)$ & $17 \%(1 / 6)$ & $23 \%(6 / 26)$ & $4 \%(1 / 26)$ \\
\hline $72 \%(34 / 47)$ & $43 \%(3 / 7)$ & $79 \%(19 / 24)$ & $8 \%(2 / 24)$ \\
\hline $35 \%(13 / 37)$ & $38 \%(3 / 8)$ & $45 \%(9 / 20)$ & $15 \%(3 / 20)$ \\
\hline $39 \%(14 / 36)$ & $17 \%(1 / 6)$ & $22 \%(6 / 27)$ & $4 \%(1 / 27)$ \\
\hline $51 \%(20 / 39)$ & $25 \%(2 / 8)$ & $45 \%(9 / 20)$ & $10 \%(2 / 20)$ \\
\hline $35 \%(11 / 31)$ & $29 \%(2 / 7)$ & $33 \%(6 / 18)$ & $11 \%(2 / 18)$ \\
\hline $58 \%(32 / 55)$ & $38 \%(3 / 8)$ & $69 \%(18 / 26)$ & $12 \%(3 / 26)$ \\
\hline $40 \%(14 / 35)$ & $17 \%(1 / 6)$ & $24 \%(6 / 25)$ & $4 \%(1 / 25)$ \\
\hline $41 \%(14 / 34)$ & $29 \%(2 / 7)$ & $35 \%(9 / 26)$ & $8 \%(2 / 26)$ \\
\hline $62 \%(31 / 50)$ & $43 \%(3 / 7)$ & $69 \%(18 / 26)$ & $0 \%(3 / 26)$ \\
\hline $45 \%$ & $28 \%$ & $43 \%$ & $8 \%$ \\
\hline
\end{tabular}

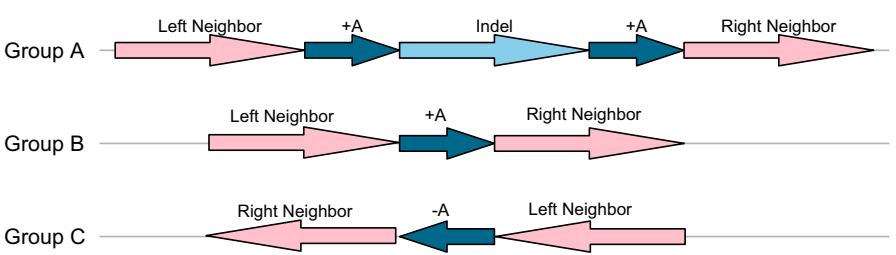

Fig. 6 Visualization of the 25 scaffolds. Each row represents the scaffold of the corresponding strain. Horizontal colored blocks are core blocks and white blocks are dispensable blocks. Each color represents a distinct set of core blocks. Integrase, transposase genes and DRs genes are represented by red, green and blue vertical bars

\section{Building scaffolds and finding instable regions}

Sequences in input genomes were segmented into core blocks, dispensable blocks and strain-specific blocks by Mugsy [8], a multiple sequence aligner. In this work, we omitted small blocks which is shorter than 1000 bp and strain-specific blocks. To 


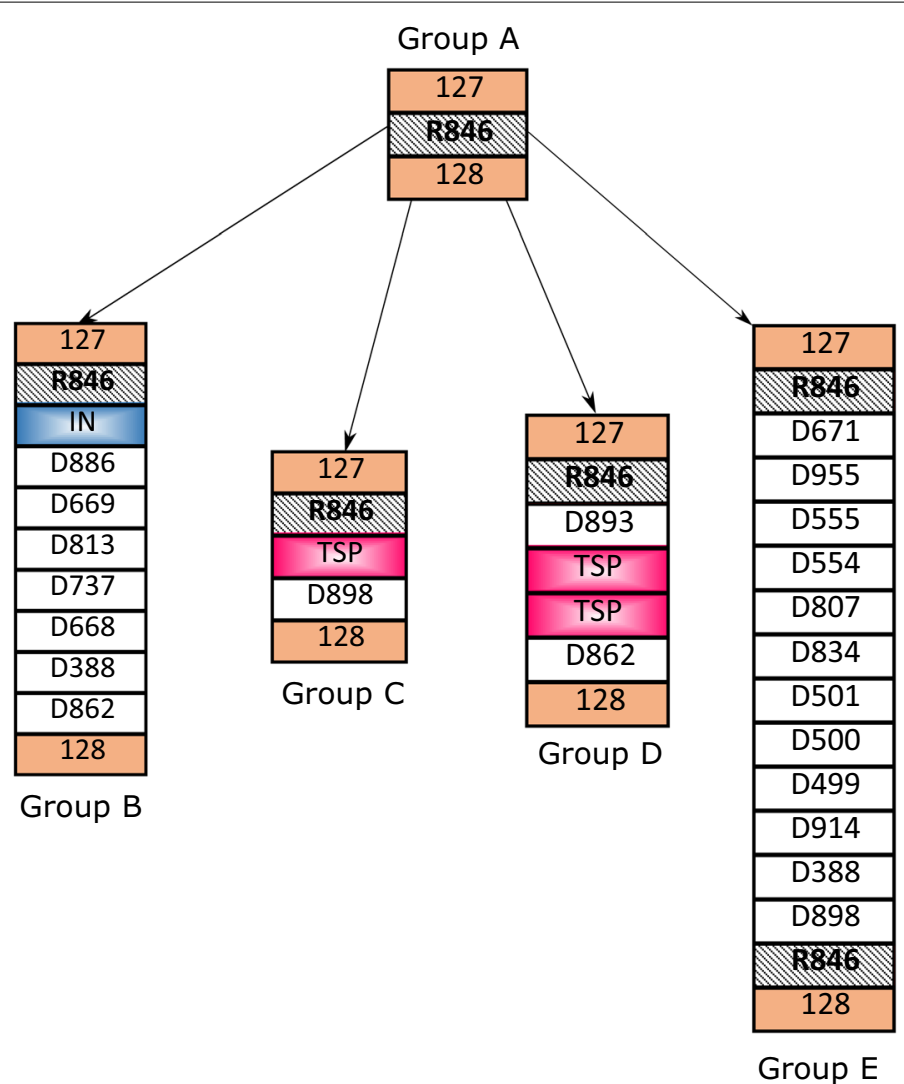

Fig. 7 Visualization of the 28 M. tuberculosis scaffolds

distinguish between different blocks, we labeled each core block with an distinct ID number and labeled each dispensable block with "D" plus an ID number. For each input genome, we then built a scaffold which consisted of the IDs of core and dispensable blocks sorted by starting positions on the chromosomes (see Additional file 1: Table S1). In the scaffolds, consecutive dispensable blocks formed instable regions and we then did a comprehensive study on these instable regions.

\section{Finding insertions and deletions mediated by DRs}

In order to figure out directed repeats that are related to instable regions, we developed a pipeline based on BLAST [9] alignment method that can find the similar structure which is shown in Fig. 8. For each instable block, act the following four procedures to find out a reasonable pair of repeats in the same direction that may be associated with the insertion or deletion of one instable region.

Firstly, regarding each instable block (define as InsB) on each strain (define as strain $A 1$ ), cut 10,000 base pairs from the left side of $\operatorname{Ins} B$ and name it as $r e A$. Define the start position of $r e A$ as $r e A \_s$ and the end position as reA_e.

Secondly, use BLAST to do alignment between reA and target segment. Target segment means the gene segment that starts from $r e A_{-} e$ and ends on the tail of strain $A 1$. The corresponding BLAST alignment outcome shows as a chart which has a set of DNA segment pairs (name this set of gene pairs as candA_list and the members in 


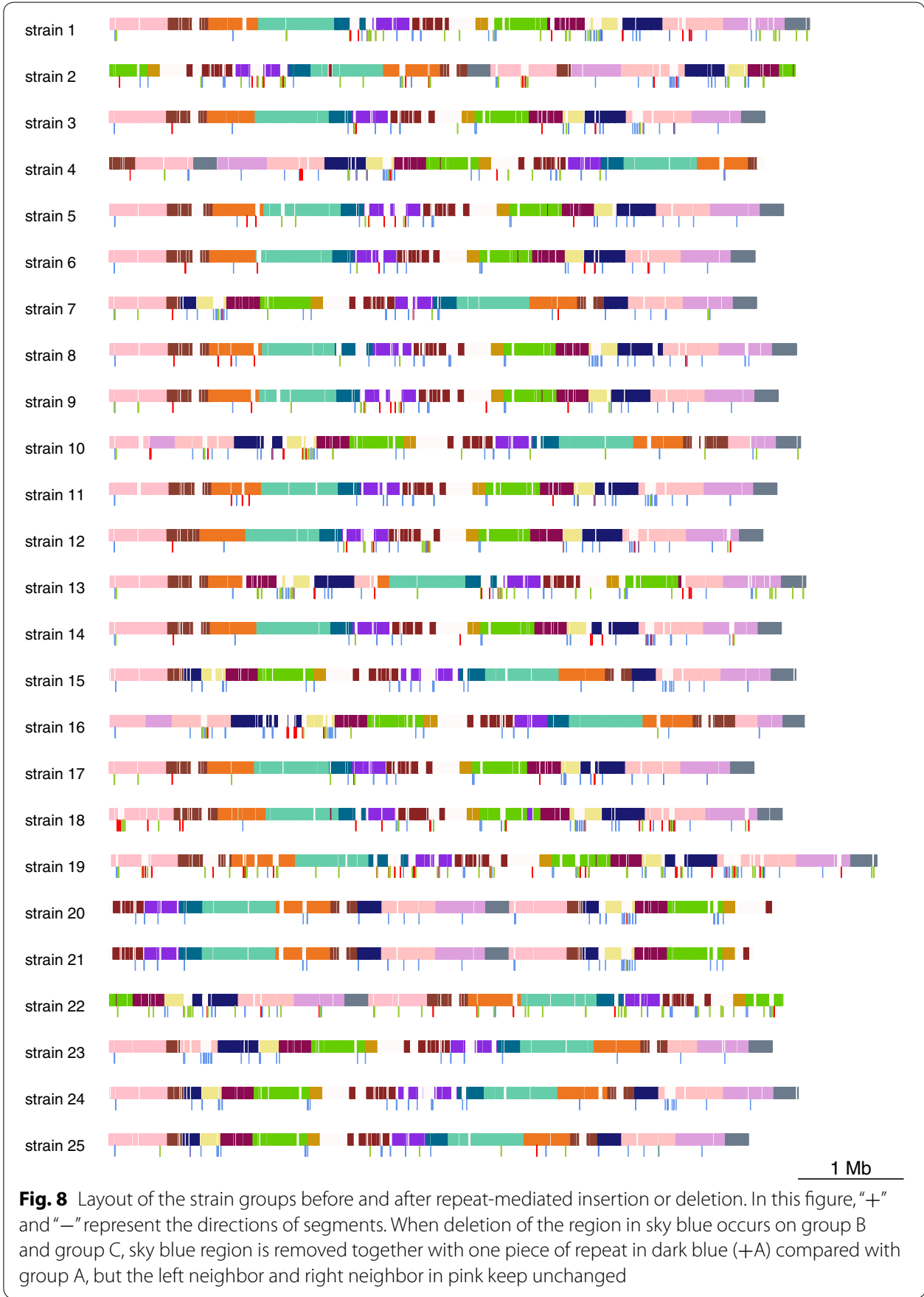

it as $c a n d A$ s), and each of them is situated at both sides of InsB, illustrating that these gene segment pairs could be the possible reason of InsB's insertion or deletion. Each line of candA_list contains four positions, left candA's start position, left candA's end position, right candA's start position and right candA's end position. We define them as candAL_s, candAL_e, candAR_s and candAR_e, respectively. For convenience, gene segment from $c a n d A L_{-} e$ to $c a n d A R_{-} s$ is named as $I N D E L$, segment from candAL_s $-10,000$ to $c a n d A L_{-} s$ is named as neighborL and segment from candAR_e to $c a n d A R_{-} e+10,000$ is named as neighborR. 
Thirdly, in order to find out which component in candA_list are linked to InsB insertion or deletion with the highest possibilities, we searched each $c a n d A$ together with its corresponding neighborL and neighborR on those strains that lack InsB. If neighborLcandA-neighborR structure can be found on those strains and neighborL-candA-InsBcandA-neighborR structure can be found on strain $A 1$ simultaneously, set this candA as RepeatA.

Lastly, for further demonstration, we check the INDEL region on all 25 strains. If INDEL region always appears together with a pair of RepeatA on both sides, RepeatA can be regarded as a reasonable repeat that causes INDEL region's insertion or deletion.

Our approach checked all the instable blocks in the .maf file and found out all the possible RepeatAs that may be the reason of gene indels. While applying BLAST package into our project, we set the E-value threshold as $10^{-10}$.

\section{Finding transposase and integrase genes}

For the two datasets, we got the positions of genes which are encoded as integrase or transposase from the annotation files in GFF format. These annotations can be accessed on NCBI. We then add these genes to their strains' scaffolds according to their positions (see Additional file 1: Tables S1 and S4).

\section{Discussion}

With the scaffolds consisting of core blocks, dispensable blocks, DRs, transposase genes and integrase genes, one can clearly observe the variations of an instable region among strains and the possible mechanisms for each variation. As illustrated in Fig. 9, the $25 P$. aeruginosa strains can be stratified into five groups (A-E) based on their instable regions between Core Block 127 and 128. Strains of Group B, C, D and E may evolve from strains of Group A through different DNA elements: the insertion of Block D886 to Block D862 in strains of Group B may be mediated by the integrase gene (blue); the insertion of dispensable block D898 in Group C may be caused by the transposase gene (deep pink); the two transposase genes (deep pink) may lead to the insertion of dispensable block D893 and D862 in the strains of Group D; the insertion of Block D671 to Block D898 in strains of Group E may be mediated by the pair of R846.

Other genetic elements, recombinations or systems, such as ICEs [10], resolvases [11], invertases [12], Illegitimate recombination [13] and CRISPR-Cas systems [14, 15], may also contribute to the variation of instable regions. In our future work, we will consider these factors to explain more variations in genomes.

\section{Conclusions}

Three characteristics of instable regions were concluded in both $P$. aeruginosa and M. tuberculosis strains: instable regions were short, site specific and varied in different strains. Three DNA elements (DRs, transposons and integrons) which may be associated with the variation of instable regions were studied. A pipeline was developed to automatically search for DR pairs on the flank of every instable sequence. We found 27 DR pairs in P. aeruginosa strains and 6 pairs in M. tuberculosis strains 


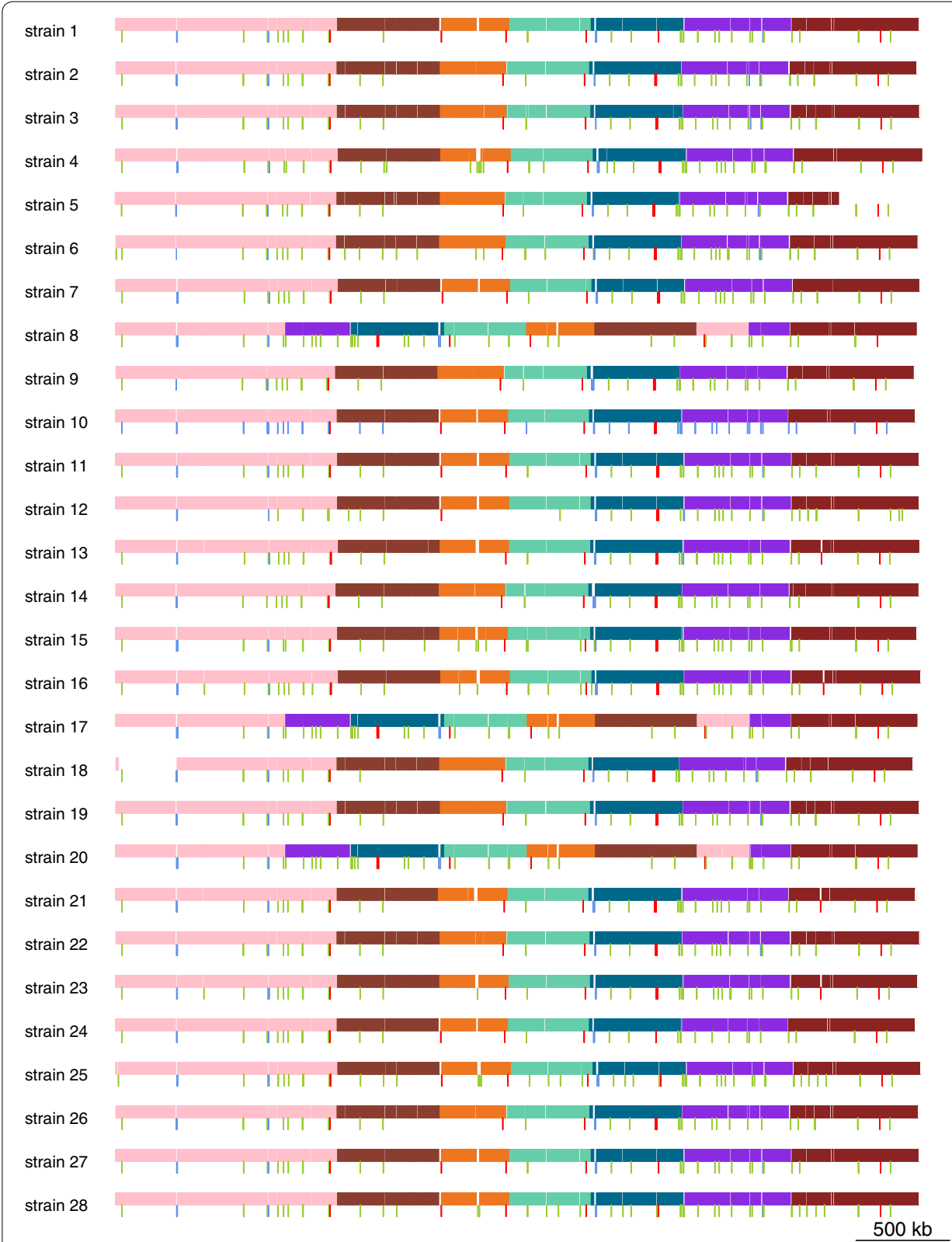

Fig. 9 Evolution of strains through different mechanisms. The five columns are the partial scaffolds (from Block 127 to 128) of strains in Group A, B, C, D and E. Core blocks, dispensable blocks, DRs, transposase and integrase genes are represented by rectangular boxes in orange, white, gray, deep pink and blue, respectively. Group A includes Strain 1, 2, 3, 5, 6, 8, 9, 10, 11, 12, 13, 14, 15, 16, 19, 22, 24 and 25. Group B includes Strain 4. Group C includes Strain 7 and 18. Group D includes Strain 17, 23. Group E includes Strain 20 and 21

existing in the instable regions. Besides, on the average, $14 \%$ and $12 \%$ of instable regions in the $25 P$. aeruginosa strains include transposase genes and integrase genes, respectively. In $M$. tuberculosis strains, an average of $43 \%$ and $8 \%$ of instable regions include transposase genes and integrase genes, respectively. 


\section{Additional file}

Additional file 1: Table S1. Sca_olds for the 25 Pseudomonas aeruginosa genomes. Table S2. The 25 Pseudomonas aeruginosa strains used for studying instable regions. Table S3. 134 distinct pairs of core blocks between which instable region existed in Pseudomonas aeruginosa strains. Table S4. Sca_olds for the 28 Mycobacterium tuberculosis genomes. Table S5. The 28 Mycobacterium tuberculosis strains used for studying instable regions. Table S6. 82 distinct pairs of core blocks between which instable region existed in Mycobacterium tuberculosis strains. Table Rn. For every achieved DR pair Rn, we showed the 25 Pseudomonas aeruginosa strains' partial sca_olds covering Rn, where $n$ is the ID of this DR pair.

\section{Abbreviations}

Tnp: transposase; IN: integrase; InsR: instable region; DR: directed repeat.

\section{Declarations}

\section{Authors' contributions}

DW designed the methods, collected the datasets, performed and analyzed the experiments, and wrote the paper. JL developed the pipeline, performed the experiments and wrote the paper. LW directed this research, designed the methods, analyzed the experimental results, and wrote the paper. All authors read and approved the final manuscript.

\section{Author details}

1 Department of Computer Science, City University of Hong Kong, 83 Tat Chee Ave., Hong Kong, People's Republic of China. ${ }^{2}$ University of Hong Kong Shenzhen Research Institute, Shenzhen Hi-Tech Industrial Park, Nanshan District, Shenzhen, People's Republic of China.

\section{Acknowledgements}

Not applicable.

\section{Competing interests}

The authors declare that they have no competing interests.

\section{Availability of data and materials}

The genome sequences and annotation files were downloaded from the NCBI Genome Database (https://www.ncbi. nlm.nih.gov/genbank/). The strains names and accession number in the NCBI Genome Database are in Additional file 1: Tables S2 and S5. Our pipeline is at: https://github.com/shever/repeat_finding.

\section{Consent for publication}

Not applicable.

\section{Ethics approval and consent to participate}

Not applicable.

\section{Funding}

Publication costs were funded by National Natural Science Foundation of China (NSFC 61373048) and a Grant from the Research Grants Council of the Hong Kong Special Administrative Region, China (CityU11256116, CityU1 14012).

\section{About this supplement}

This article has been published as part of BioMedical Engineering OnLine Volume 17 Supplement 1, 2018: Selected articles from the 5 th International Work-Conference on Bioinformatics and Biomedical Engineering: biomedical engineering. The full contents of the supplement are available online at https://biomedical-engineering-online.biomedcentral.com/ articles/supplements/volume-17-supplement-1.

\section{Publisher's Note}

Springer Nature remains neutral with regard to jurisdictional claims in published maps and institutional affiliations.

Published: 20 November 2018

\section{References}

1. Tettelin H, Masignani V, Cieslewicz MJ, Donati C, Medini D, Ward NL. Genome analysis of multiple pathogenic isolates of Streptococcus agalactiae: implications for the microbial "Pan-Genome". Proc Nat Acad Sci UA. 2005;102(39):13950-5.

2. Vernikos G, Medini D, Riley DR, Tettelin H. Ten years of Pan-Genome analyses. Curr Opin Microbiol. 2015;23:148-54.

3. Song $\mathrm{H}$, Hwang J, Yi H, Ulrich RL, Yu Y, Nierman WC. The early stage of bacterial genome-reductive evolution in the host. Plos Pathog. 2010;6(5):E1000922.

4. Marsh JW, O'leary MM, Shutt KA, Harrison LH. Deletion of feta gene sequences in Serogroup B And C Neisseria meningitidis isolates. J Clin Microbiol. 2007;45(4):1333-5.

5. Darmon E, Leach DR. Bacterial genome instability. Microbiol Mol Biol R. 2014;78(1):1-39.

6. Radding C. Homologous pairing and strand exchange promoted by Escherichia coli reca protein. Washington, DC: American Society For Microbiology; 1988. p. 193-229. 
7. Polard P, Chandler M. Bacterial transposases and retroviral integrases. Mol Microbiol. 1995;15(1):13-23.

8. Angiuoli SV, Salzberg SL. Mugsy: fast multiple alignment of closely related whole genomes. Bioinformatics. 2011;27(3):334-42.

9. Tatusova TA, Madden TL. Blast 2 sequences, a new tool for comparing protein and nucleotide sequences. Fems Microbiol Lett. 1999;174(2):247-50.

10. Miguel $B$, et al. Integrating conjugative elements of the $S \times t / R 391$ family from fish-isolated vibrios encode restriction-modification systems that confer resistance to bacteriophages. Fems Microbiol Ecol. 2013;83(2):457-67.

11. Brassard S, Paquet H, Roy PH. A transposon-like sequence adjacent to the acci restriction-modification operon. Gene. 1995;157(1):69-72.

12. VaiŠVila R, Vilkaitis $G$, Janulaitis A. Identification of a gene encoding a DNA invertase-like enzyme adjacent to the Paer7i restriction-modification system. Gene. 1995;157(1):81-4.

13. Ehrlich S, Bierne H, D'alencon E, Vilette D, Petranovic M, Noirot P, et al. Mechanisms of illegitimate recombination. Gene. 1993;135(1):161-6.

14. Sorek R, Kunin V, Hugenholtz P. Crispr-A widespread system that provides acquired resistance against phages in bacteria and archaea. Nat Rev Microbiol. 2008;6(3):181-6.

15. Van Der Oost J, Jore MM, Westra ER, Lundgren M, Brouns SJ. Crispr-based adaptive and heritable immunity in prokaryotes. Trends Biochem Sci. 2009;34(8):401-7.

- fast, convenient online submission

- thorough peer review by experienced researchers in your field

- rapid publication on acceptance

- support for research data, including large and complex data types

- gold Open Access which fosters wider collaboration and increased citations

- maximum visibility for your research: over $100 \mathrm{M}$ website views per year

At BMC, research is always in progress.

Learn more biomedcentral.com/submissions 